\section{The central source in the supernova remnant G127.1 + 0.5}

THE discovery ${ }^{1}$ of a very small diameter radio source with a very fiat spectrum at the centre of the galactic supernova remnant $\mathrm{G} 127.1+0.5$ has stimulated much interest $\mathrm{t}^{2-4}$. Because the central source has unusual properties, and because the a priori probability of finding such an object at the very centre of a supernova remnant is exceedingly low, Caswell ${ }^{1}$ suggested that the central radio source might be emanating from the collapsed stellar remnant of the supernova that produced the surrounding shell of radio emission. This intriguing idea was pursued by Salter, Pauls and Halsam ${ }^{3}$ who showed that the extended supernova remnant is perfectly normal in shape and in radio spectrum, while the central source has a spectrum that is flat from 3 to $15 \mathrm{GHz}$ at a flux density of $0.5 \mathrm{Jansky}$. A long-baseline interferometric measurement by Shaffer et al. ${ }^{4}$ at $10.65 \mathrm{GHz}$ showed that the angular size of the central source is about 0.0005 arcs. We now present evidence from direct optical observation suggesting that the link between the central source and the supernova remnant is purely circumstantial.

Caswell suggested, based on positional coincidence to 0.5 arc $s$, that a red object of about 19 mag was identical with the source of radio emission. Inspection of the Palomar Sky Survey shows that the object is diffuse on the red plate, and invisible on the blue plate. The entire region near $\mathrm{G} 127.1+0.5$ shows signs of heavy obscuration.

We have made spectrophotometric measurements of the red diffuse object with the image dissector scanner at the 4-m Mayall telescope of Kitt Peak National Observatory. Our observations were obtained on 28-29 August 1978, a night of excellent seeing and transparency. On the slit-viewing television, the object was clearly nonstellar. We integrated for $4,500 \mathrm{~s}$ and obtained simultaneous object and sky spectra through two apertures of diameter 3.2 arc s separated by 52 arc s. The net spectrum due to the object covered the wavelength interval 4,000-7,500 $\AA$ with a resolution of about $20 \AA$. Figure 1 shows the distribution of flux density $f_{\lambda}$ in erg cm $\mathrm{cm}^{-2} \mathrm{~s}^{-1} \AA^{-1}$ obtained by comparing the counts observed on G127.1+0.5 with those obtained on standard stars of known flux distribution.

As Fig. 1 shows, the spectrum of the object consists of a very red continuum with two broad emission blends. The two blends

Fig. 1 Flux density $F_{\lambda}\left(\mathrm{erg} \mathrm{cm}^{-2} \mathrm{~s}^{-1} \AA^{-1}\right)$ plotted against wavelength for the diffuse red object at the centre of the supernova remnant $\mathrm{G} 127.1+0.5\left(\alpha=01\right.$ h $25 \mathrm{~min} 08.3 \mathrm{~s}, \delta=+62^{\circ} 50^{\prime} 59^{\prime \prime}$ (1950)). The two broad emission features at $6,691 \AA$ and at $6,841 \AA$ are identified with [N $\mathrm{II}]+\mathrm{H} \alpha$ and with [S II] at a redshift of $5,375 \pm 200 \mathrm{~km} \mathrm{~s}^{-1}$

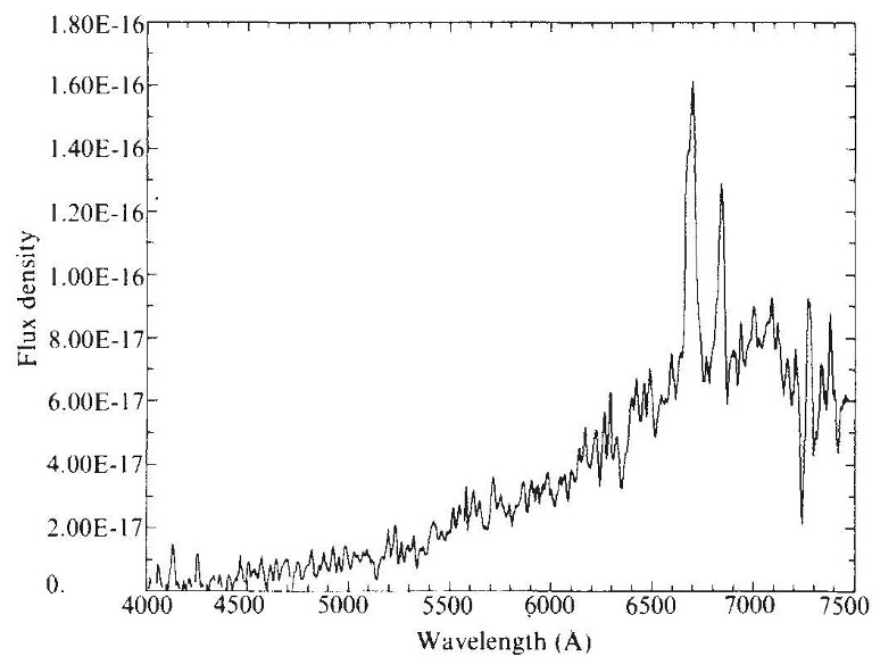

are at wavelengths of $6,691 \AA$ and $6,841 \AA$, and have integrated fluxes in the ratio $2.3: 1$. We identify the stronger, broader feature at $6,691 \AA$ with the familiar blend of [N II] $6,548.1 \AA$, $\mathrm{H} \alpha \quad 6,562.8 \AA$, and $[\mathrm{N} \mathrm{II}] 6,583.6 \AA$. The feature observed at $6,841 \AA$, we identify with the [S II] doublet $6,717.0,6,731 \AA$, and we propose that the object is a galaxy.

Although the individual lines are not resolved by our instrument, we can make a reasonable estimate for the relative line strengths within the blends by using the spectrophotometry of typical radio galaxies compiled by Costero and Osterbrock ${ }^{5}$. Based on their work, we expect that [N II] $6,584 \AA$ will have about the same strength as $\mathrm{H} \alpha$, and that [N II] $6,548 \AA$ has one-third that strength. We expect that the [S II] $6,717 \AA$ line will have the same strength as the $6,731 \AA$ line. On that basis we compute effective wavelengths for the blends that yield a redshift $c z=5,375 \pm 200 \mathrm{~km} \mathrm{~s}^{-1}$. We note that the observed ratio of $[\mathrm{N} \mathrm{III}]+\mathrm{H} \alpha$ to $[\mathrm{S} \mathrm{II}]$ is about the same in this object as in the radio galaxies studied by Costero and Osterbrock.

If the object is at a distance of $\sim 100 \mathrm{Mpc}$, (for $H_{0}=$ $55 \mathrm{~km} \mathrm{~s}^{-1} \mathrm{Mpc}^{-1}$ ) then it has an absolute magnitude $M_{\mathrm{v}}$ of about $-16-A_{\mathrm{v}}$. We can estimate the absorption $A_{\mathrm{v}}$ by noting that the continuum fluxes at 7,000 $\AA$ and $5,800 \AA$ are in the ratio $2.7: 1$. Absolute spectrophotometry by Oke and Sandage ${ }^{6}$ indicates that the intrinsic ratio is about $1: 1$. If we attribute the reddening to dust that follows the Whitford relation ${ }^{7.8}$ the required extinction at the visible is $A_{v}=5 \mathrm{mag}$. Then the absolute magnitude of the object is near $M_{\mathrm{v}}=-21$, the absolute magnitude of a luminous galaxy. The presence of large extinction is corroborated by the low galactic latitude of the source $\left(b=+0.5^{\circ}\right)$, the appearance of the region on the Palomar Sky Survey, the absence of the object from the blue plate, and the absence of short wavelength emission lines, such as [O III] 5,007 $\AA$, that are prominent in radio galaxies.

At a distance of $100 \mathrm{Mpc}$, the radio power of the galaxy integrated to $15 \mathrm{GHz}$ is about $8 \times 10^{40} \mathrm{erg} \mathrm{s}^{-1}$, coming from a region of diameter $0.2 \mathrm{pc}$. For comparison, the compact source in M 87 has a diameter of $0.04 \mathrm{pc}$, and about $\frac{1}{8}$ the emitted energy per unit frequency at $15 \mathrm{GHz}$ (ref. 9).

We believe that a completely consistent picture of the central source in G127.1+0.5 is that it is a luminous galaxy at a distance of $\sim 100 \mathrm{Mpc}$ that contains a compact and energetic radio source. Although the circumstantial evidence linking the central source to the supernova remnant is intriguing, we believe that the positional coincidence is mere chance.

Because G127.1 + 0.5 was the most compelling case for a compact radio source associated with a supernova remnant, the demonstration that this object is a chance event makes it seem likely that other suggested radio objects near supernova remnants may well be just the superposition of background sources on galactic remnants.

Kitt Peak National Observatory is operated by Aura, Inc., under NSF contract. R.P.K. was supported by NSF grant AST 77-17600.

ROBERT P. KIRSHNER

Department of Astronomy,

University of Michigan,

Ann Arbor, Michigan 48109

Roger A. Chevalier

Kitt Peak National Observatory,

Tucson, Arizona 85726

Received 11 September; accepted 18 October 1978.

1. Caswell, J. L. Mon. Not. R. astr. Soc. 181, 789-797 (1977).

2. Pauls, T. Astr. Astrophys. 59, L13-14 (1977).

3. Salter, C. J., Pauls, T. \& Halsam, C. G. T. Astr. Astrophys. 66, 77-80 (1978)

4. Shaffer, D. B. et al. Astr. Astrophys. 68, L11-13 (1978).

5. Costero, R. \& Osterbrock, D. E. Astrophys. J. 211, 675-683 (1977).

6. Oke, J. B. \& Sandage, A. R. Astrophys. J. 154, 21-32 (1968).

7. Whitford, A. E. Astr. J. 63, 201-207 (1958).

8. Mitler, J. S. \& Mathews. W. G. Astrophys. J. 172, 593-607 (1972).

8. Miller, J. S. \& Mathews. W. G. Astrophys. J. 172, 593-607
9. Kellermann, K. I. et al. Astrophys. J. 211, 658-668 (1977), 